\title{
NANOGAP ULTRASONIC ACTUATOR FOR NON-CONTACT CONTROL OF LEVITATED INERTIAL SENSOR ROTOR
}

\author{
S. Piratla ${ }^{*}$ M. Pandey, and A. Lal
}

SonicMEMS Laboratory, School of Electrical and Computer Engineering, Cornell University, USA

\begin{abstract}
In this paper we present an ultrasonic motor with a rotor which levitates creating an air bearing with smooth rotation at 100 $600 \mathrm{rpm}$, whose motion can be controlled without contact. We demonstrate the use of a thermal actuator-driven gripper-actuator that can control the speed and direction of the rotor, without contact, while it is levitated. This same actuator can also be used to stop the rotor by direct mechanical contact. Voltages required for this actuation are in the 1-10 Volts, making the calibration platform CMOS compatible. In order to demonstrate the application of this rotor for inertial sensor calibration, we demonstrate the first integration of optically read accelerometers, read out while the rotor is spinning.
\end{abstract}

\section{KEYWORDS}

Ultrasonic, micromotor, piezoelectric, thermal actuator, levitation, accelerometer, diffraction, optical readout

\section{INTRODUCTION}

One of the most challenging problems for reaching ideal MEMS inertial sensor performance is the change in bias and scale factor over time, and over environmental variables such as shock, temperature, humidity and packaging. Several attempts have been made to eliminate bias by making individual mechanical and electrical components of the MEMS inertial sensors symmetric and higher Q. However, with limits on precision of fabrication steps such as lithography and etching, and spatial variation of material properties of thin films, bias elimination remains a hard challenge. One way to eliminate biases is to calibrate the biases actively in the sensor package itself. Micro scale stable rotating platforms with integrated inertial sensors hold the promise of performing in-situ gyroscope and accelerometer sensor calibration by applying known accelerations and rotation rates. The biases can be measured by measuring signals with sensors at known angles (Figure 1).

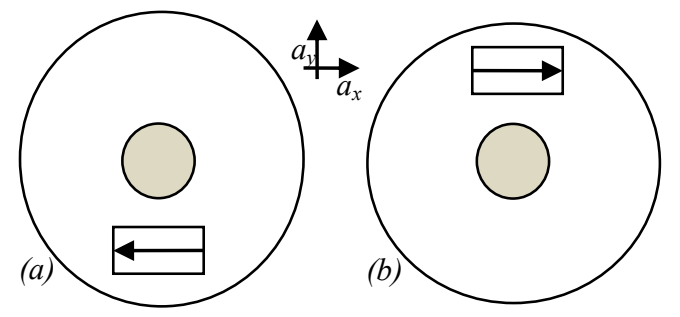

Figure 1.Calibration of $x-y$ accelerometers on a rotatable platform. In (a) the accelerometer sensitivity is negative of (b) - enabling the extraction of bias $V_{b}$ in the accelerometer measured voltage $V_{a x}=S_{a} a_{x}+V_{b}$.

However, for proper calibration of the inertial sensor scale factor the rotation of the platform needs to be free of secondary features that can complicate sensor signals. For example, a rotating platform needs to rotate smoothly without actuator artifacts such as jerks. Hence, it is critical to rotate the sensor without any physical contact. This is equivalent to suspending the rotor during calibration.
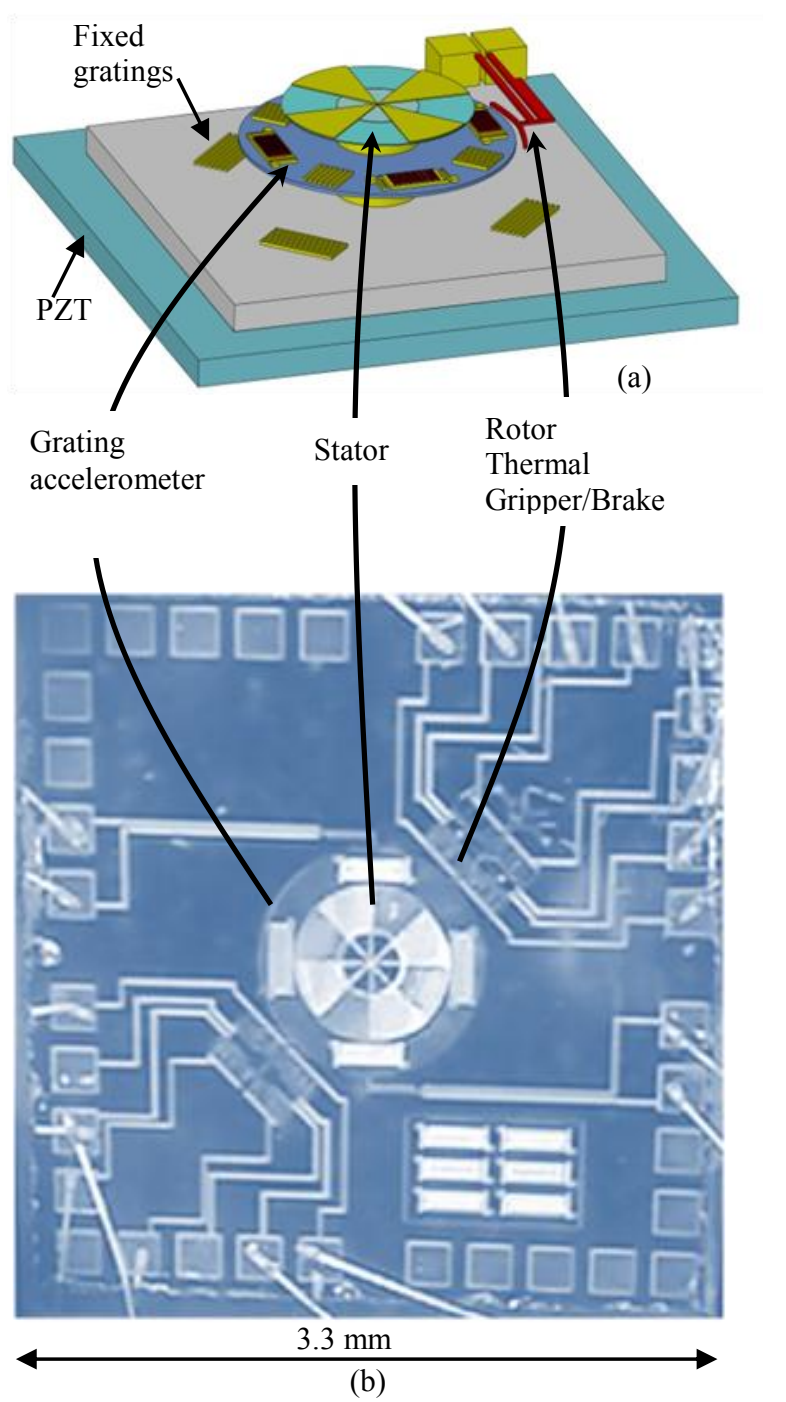

Figure 2. (a) Schematic of ultrasonically driven motor with integrated grating accelerometer. A thermal actuator is used to move a gripper close to the spinning levitated motor.

(b) Optical micrograph of wire bonded device

Many previous proof of principles exist for suspending rotating platforms. Magnetic suspension has been shown using diamagnetic rotors [1],[2]. In magnetic case, high currents are typically needed to generate sufficient magnetic field gradients for levitation. Electrostatic suspension has been achieved in implementing gyroscope itself by several investigators [3],[4]. 
In electrostatic case, relatively high voltages and vacuum are needed to achieve flotation and vacuum operation. An alternative to electromagnetic forces is to use fluidic forces to suspend rotating platforms. An alternative to suspended operation is to use viscous drag forces induced by acoustic and dynamical forces. By actuating a plate at its ultrasonic resonant modes, the resulting acoustic streaming and acoustic radiation forces can be used to levitate the rotor.

\section{PRINCIPLE OF OPERATION}

Figure 2 shows the overall concept of the inertial sensor calibration platform, implemented in MUMPS process. The rotor has thickness of $2 \mu \mathrm{m}$ and a diameter of $900 \mu \mathrm{m}$. The bulk PZT bonded to the motor is excited at amplitude of $10 \mathrm{Vpp}$ near resonant frequencies of the hub-stator, some of which are whispering gallery modes. These modes have lower air damping and are thus observed in air at high amplitudes. The rotating mode couples energy from stator to rotor through an air-fluid bearing via acoustic streaming, which is a time averaged steady flow of the fluid that usually happens due to varying acoustic intensity in the fluid medium. The resulting drag force pushes the rotor, which we have reported in our earlier work [5],[6]. Recently we optimized the acoustic coupling by programming gap as a function of theta by adding gold coverage on the stator [6],[7]. The built-in-stress in gold results in bending of the stator and rotor, giving rise to a periodic variation of stator-rotor gap, $g(\theta)=a+b^{*} \sin (n \theta)$, where a is $8.6 \mu \mathrm{m}$ and $\mathrm{b}$ is $5.8 \mu \mathrm{m}$ for $\mathrm{n}=2$. The bending of the stator allows for more space to help with the levitation.

Thermal and electrostatic actuators shown in Figure 2 can move grippers towards the spinning rotor to hold it in place during sensor operation, or control its rotation direction and rate. Accelerometers and gyros can be integrated onto the rotor, and in Fig. 2 we show an optical grating accelerometer that changes the grating spacing due to accelerations. In this paper we demonstrate non-contact operation from levitation, and also non-contact control of rotation from the grippers. We can also control the direction and rotation without contact.

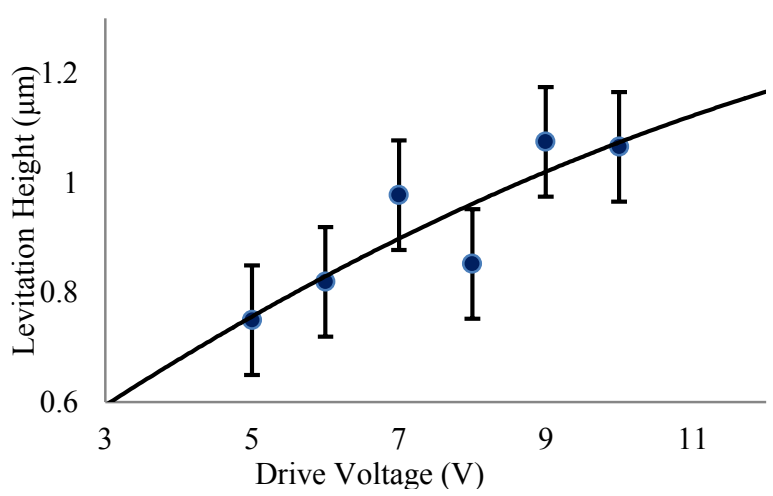

Figure 3: Variation of levitation height during rotation (measured with ZYGO) with drive voltage at $3.25 \mathrm{MHz}$. The stable levitation is at $1 / 2$ of rotor-stator gap.

\section{LEVITATION}

Levitation in fluid based systems can be achieved due to pressure built-up as a result of periodic forcing of the fluid. Here, resonant actuation of the substrate launches pressure wave in the air gap, with a wavelength $\sim 60 \mu \mathrm{m}$, which is larger than the gap between the stator and the base (about $1 \mu \mathrm{m}$ ). Hence squeeze film levitation is dominant; where periodic squeezing forces the fluid to flow out through the sides but the shear resistance prevents it.
This gives effective stiffness to the trapped air region. The resulting levitation force from a Reynolds flow based analysis is estimated by [8]:

$W=\frac{5 \varepsilon^{2}}{4 \beta}\left[\frac{\beta \cos (\beta)+\beta \cosh (\beta)-\sin (\beta)-\sinh (\beta)}{\cos (\beta)+\cosh (\beta)}\right]$

where $\beta=\sqrt{\frac{\sigma}{2}}$ with $\sigma=\frac{12 \omega \mu L^{2}}{p_{a} h_{0}^{2}}$ being the squeeze number, $\omega$ the drive frequency, the viscosity, $\mathrm{L}$ the diameter, $\mathrm{p}_{\mathrm{a}}$ the ambient pressure and $\mathrm{h}_{0}$ the initial gap.

We demonstrate that during rotation the rotor levitates and stays biased in the middle of the stator and the substrate. The larger stator deflection leads to more space for the rotor to levitate. The levitation is a function of rotation rate (rate function of drive voltage) as expected due to rotation induced lift force on the rotor (Figure 3). This compares well with the analytical results.

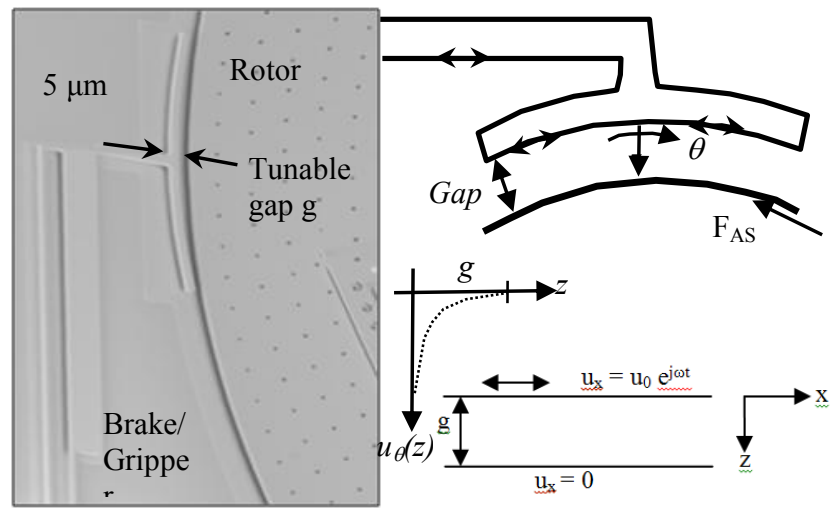

Figure 4: SEM of thermal actuator that moves the gripper towards rotor. Other structures on chip are for other testing not important to abstract. Gripper moves due to substrate motion and creates gradient in a sonic shear viscous field $\left(u_{\theta}\right)$ giving rise to acoustic streaming force $F_{A S}$.

\section{THERMAL ACTUATOR GRIPPER}

This is a thermal bimorph actuator to control the speed and direction of the rotor (Figure 4). The gripper actuator, anchored to the substrate, also moves at the PZT drive frequency.

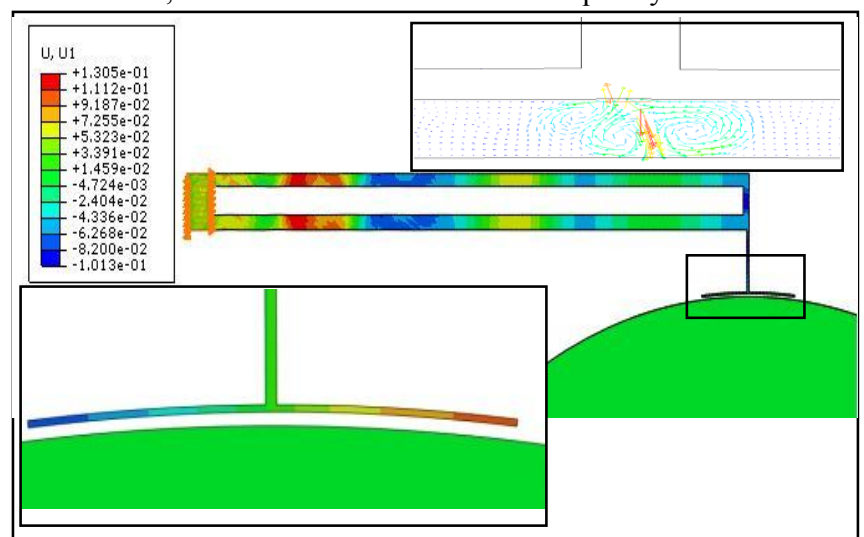

Figure 5. Ultrasonic motion of the brake pad as a function of forcing and resulting streaming.

The motion creates a gradient of velocity between gripper and rotor. This shear viscous field (shear viscous depth $0.9 \mu \mathrm{m}$ at $3.25 \mathrm{MHz}$ ) gives rise to a time averaged acoustic streaming driven force (Figure 4) [4]. Torque on the rotor is obtained from the following analysis. 
We solve the Navier-Stokes equation in the gap

$$
\rho \frac{\partial u}{\partial t}+\rho u \cdot \nabla u=f-\nabla p+\mu \nabla^{2} u
$$

As $u \cdot \nabla u$ is a second order term, it can be neglected.

No forces: $\mathrm{f}=0$

No pressure difference: $\nabla p=0$

Under these assumptions the above equation reduces to

$$
\rho \frac{\partial u}{\partial t}=\mu \frac{\partial^{2} u_{x}}{\partial z^{2}}
$$

The solution to the above equation is

$$
u_{x}=u_{0}\left(A e^{\frac{-z}{\delta}}+B\right) e^{j \omega t}
$$

Where

$\delta=\sqrt{\frac{\mu}{2 \rho \omega}}$ is the thickness of shear viscous layer.

With boundary conditions

$u_{x}=\left.u_{0}\right|_{\mathrm{z}=0}$ and $u_{x}=\left.0\right|_{\mathrm{z}=\mathrm{g}}$

we get $A=\frac{u_{0} e^{\frac{g}{2 \delta}}}{2 \sinh \left(\frac{g}{2 \delta}\right)}, B=-\frac{u_{0} e^{\frac{g}{2 \delta}}}{2 \sinh \left(\frac{g}{2 \delta}\right)}$

The resulting Reynolds stress is calculated from [8]

$$
f=\left\langle u \frac{\partial u}{\partial z}\right\rangle
$$

The total force is then obtained by integrating this term over the entire gap.

Hence the total force $=L \cdot W \cdot \int_{0}^{g}\left(u \frac{\partial u}{\partial z}\right) d z=$ W. h. $\Gamma\left(\frac{g}{\delta}\right)$

Where

$$
\Gamma\left(\frac{g}{\delta}\right)=-\frac{2 u_{0}^{2} \sin \left(\frac{g}{\delta}\right)}{4 \sinh ^{2}\left(\frac{g}{2 \delta}\right)}-\frac{u_{0}^{2}}{4 \sinh ^{2}\left(\frac{g}{2 \delta}\right)}\left(e^{-\frac{g}{\delta}}-1\right)
$$

The torque can be calculated as

$$
T=\vec{r} \times \vec{F}=R . W . h . \Gamma\left(\frac{g}{\delta}\right)
$$

The rotation rate of the rotor should be proportional to the torque. Using the expression of the torque from Eq.5 and using the values of the shear viscous depth at $3.8 \mathrm{MHz}$, we calculated the modified the rotation rate to be:

$$
\omega=\omega_{0}+A * T
$$

Where $\omega_{0}$ is the rotation rate due to the stator torque, and is the rate when the gripper-rotor gap is large with minimal effect on the rotation rate. As the gap decreases, the gripper toque increases. As can be seen in Figure 6, Eq.8 fits very well up to maximum rotation rate. After the peak in rotation rate, the equation does not fit, indicating different dynamics. Most likely, the gripper-rotor gap becomes small enough to periodically touch the rotor, resulting in periodic holding, culminating in reduced rotation rate. This part of the motion can also be modeled.

This fluidic torque from the thermal actuator can add to or counteract the acoustic streaming torque from the stator drive. If the shear viscous force due to thermal actuator gripper is in $\mathrm{CW}$ (clockwise) direction, and if the rotor is rotating in $\mathrm{CW}$ direction due to stator force, the forces add constructively and we observe an increase in rotation rate of the rotor as the gripper - rotor gap decreases (Figure 6). On the other hand, if the rotor is rotating in $\mathrm{CCW}$ (counter clockwise) direction, the rotation rate decreases as the gripper - rotor gap decreases eventually making the rotation rate zero and then reverses the direction of the rotation to $\mathrm{CW}$ (Figure 7).

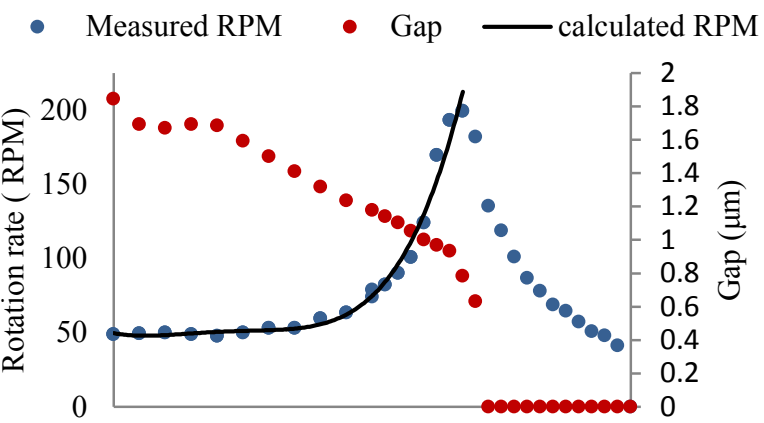

Figure 6: Rotation rate increases as gripper gap decreases with increasing actuator voltage (Figure 3 expressions predict the increase as shown). As the gripper starts touching the rotor, the rotation rate decreases.

As the drive voltage for the PZT increases $\left(12.2-12.6-13 \mathrm{~V}_{\mathrm{pp}}\right.$ in Figure 7), the stator force and the gripper force both increase since the PZT drive is the source of energy for both actuators. As the PZT drive voltage increases, the transition from $\mathrm{CW}$ to $\mathrm{CCW}$ occurs at lower gaps. This could be due to better coupling of motion to the gripper, compared to the stator. The seeming variability of direction and amplitude near the transition point (Figure 7) could be due to the unsteady contact dynamics between the rotor and the gripper. The gripper force is sufficient to drive the rotor in opposite direction, indicating that the torques generated by the stator and the gripper are comparable. At higher gripperactuator voltages, one end of the gripper touches the rotor, decreasing the rotation rate due to friction and eventually clamping it (Figure 6).

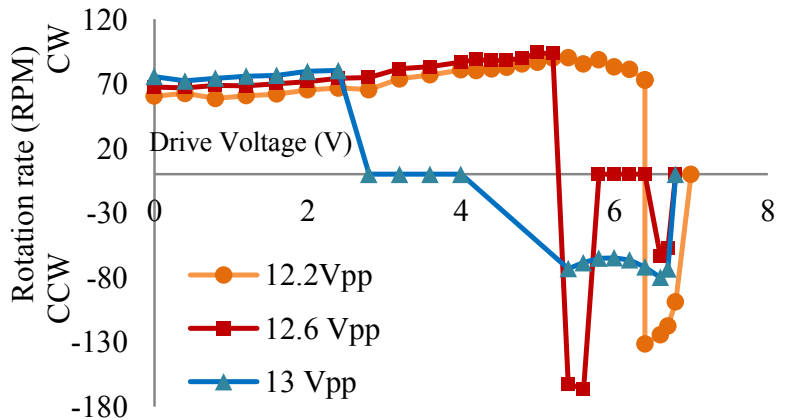

Figure 7: Rotation rate varying with thermal actuator voltage shows that shear viscous force can change direction of rotation $(C C W-$ to $C W+)$. Threshold changes with drive voltage, with increased role of shear drive.

Upon illumination from a $650 \mathrm{~nm}$ laser VCSEL diode (Thorlabs L650P007), the diffraction pattern was recorded and digitally analyzed. The diffraction pattern from G1 and G2 are orthogonal to each other and can be used to calibrate the expected and deviations of the pattern from the accelerometer. We can measure the change in the accelerometer grating response as a function of rotation rate (Figure 8). By bending of the diffraction grating, we can see that the moving grating fingers are most likely getting twisted by radial acceleration due to rotation rate. 


\section{SELF-CALIBRATING DIFFERENTIAL OPTICAL ACCELEROMETER}

A preliminary optical grating accelerometer was implemented on the rotor with built-in self-calibrating gratings. The accelerometer consists of a set of grating reflecting fingers implemented in MUMPS gold that move in circumferential direction as a result of acceleration with springs. In the middle of the moving fingers are fixed gold reflecting lines. The accelerometer provides a diffraction pattern recorded by a CMOS imager, with interference between light reflecting from the accelerometer (AG), while there are fixed gratings (G1 and G2) (Figure 8(a)).

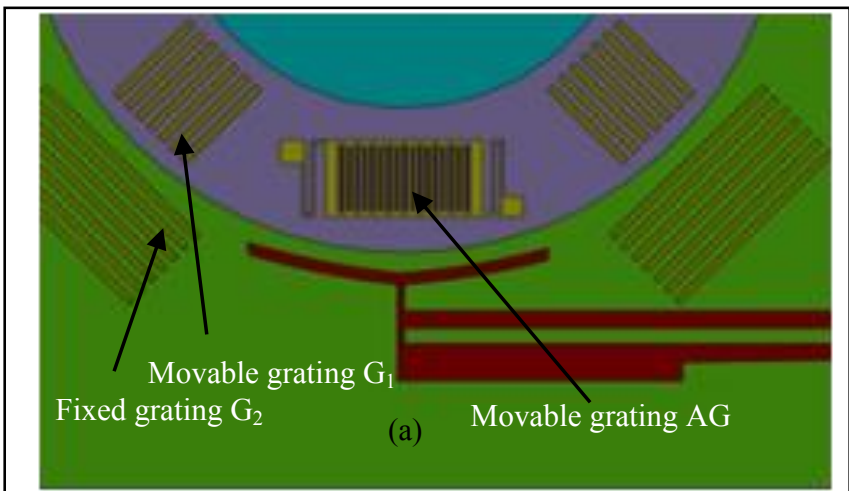

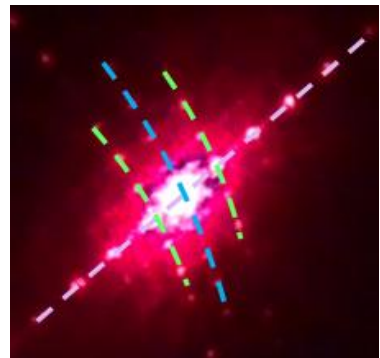

(b)

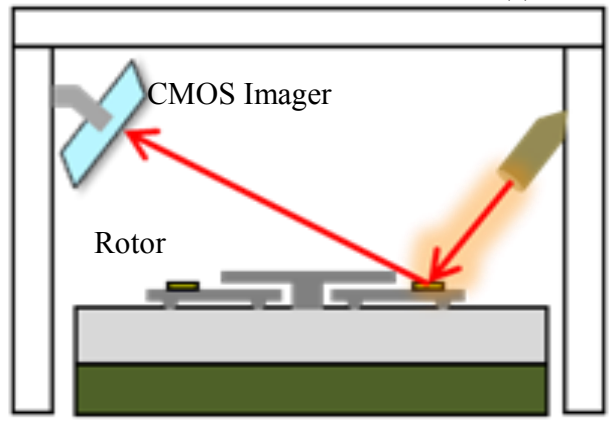

(d)

Figure 8: (a) fixed and moving gratings (b) \& (c) shows diffraction pattern changing due to rotor motion (d) Showing ultrasonic motor, VCSEL, CMOS imager for optical read out of accelerometer in a package of $10 \times 2 \times 3 \mathrm{~cm}$.

\section{CONCLUSIONS}

We have outlined a generic platform for in-situ calibration of inertial sensors, using a rotor that can be positioned at any angle and also be actuated to provide stimulus to calibrate the sensor bias and scale-factor respectively. In order to accomplish calibration, we demonstrate building blocks of a platform that do this without motion artifacts by control in levitated state. We have demonstrated that using acoustic radiation forces we can levitate spinning rotor, actuated by acoustic streaming forces. The levitation leads to rotor stabilized to middle of the gap between a stator and substrate. We have also implemented a thermal gripper that serves two purposes. With zero gap between the rotor and the gripper, the gripper acts to hold the rotor in place. By adjusting the gap to sub-micron level, we can control the rotation rate and direction of the rotation. Acoustic streaming based model of torque generation due to relative motion between the gripper and the rotor at ultrasonic frequencies is developed, which agrees with measured data. Further works needs to combine the control with integrated inertial sensors, as outlined with preliminary work on an optical grating accelerometer.

\section{ACKNOWLEDGEMENTS} support.

The authors would like to thank DARPA for financial

\section{REFERENCES}

[1] C. Shearwood, K. Y. Ho, C. B. Williams, "Development of a levitated micromotor for application as a gyroscope," Sensors and Actuators A, vol. 83, pp. 85-92, 2000.

[2] M. Kraft, M. M. Farooqui, A. G. R. Evans, "Modeling and design of an electrostaticallv levitated disk," J. Micromech Microeng, vol. 11, no. 4, pp. 423-427, 2001.

[3] M. Takao,E.Yasuo; F.Keisuke,N. Sigeru; E.Masayoshi ,'Electrostatically levitated ring-shaped rotationalgyrol/accelerometer", Japanese Journal of Applied Physics, Part 1: Regular Papers and Short Notes and Review Papers, v 42, n 4 B, p 2468-2472, April 2003.

[4] B.Dillard,V.Trent, M.Greene, E. W.Taylor, "Radiation effects on multiple DOF MEMS inertial sensors", Proceedings of SPIE - The International Society for Optical Engineering, v 8164, 2011, Nanophotonics and Macrophotonics for Space Environments $V$.

[5] V.Kaajakari, A. Lal, "Micromachined Ultrasonic Motor based on Parametric Polycrystalline Silicon Plate Excitation", Sensors and Actuators A: Physical, Vol137, No.1, pp120-128, June 2007.

[6] M. Pandey, A. Lal, "Analysis of Ultrasonic Motor in air using Fluid Structure Interaction Based Simulations", Ultrasonics 2011, Orlando Fl.

[7] S. Piratla, A. Lal, "Resonant Stator Actuation of Gear Coupled Ultrasonic Motors", Transducers 2011, Beijing, pp 2450-2453.

[8] A. Minikes and I. Buche "Levitation force induced by pressure radiation in gas squeeze films", J. Acoust. Soc. Am., Vol. 116, No. 1, July 2004.

[9] W. L. Nyborg"Acoustic Streaming near a Boundary", The Journal Of The Acoustical Society Of America, Volume 30, Number 4 April, 1958.

\section{CONTACT}

*S.Piratla, sp748@cornell.edu 